\title{
Applications of a picture fuzzy correlation coefficient in pattern analysis and decision-making
}

\author{
Surender Singh ${ }^{1} \cdot$ Abdul Haseeb Ganie $^{1}$ (B) \\ Received: 15 March 2021 / Accepted: 3 June 2021 / Published online: 12 July 2021 \\ (C) The Author(s), under exclusive licence to Springer Nature Switzerland AG 2021
}

\begin{abstract}
Picture fuzzy set is an efficient tool for dealing with uncertainty and vagueness, particularly in situations that require assimilation of more dimensions of linguistic assessment such as human voting, feature selection, etc. The correlation coefficient of picture fuzzy sets is a tool to determine the association of two picture fuzzy sets. It has several applications in various disciplines like science, engineering, and management. The prominent applications include decision-making, pattern recognition, clustering analysis, medical diagnosis, etc. In this paper, we introduce a new correlation coefficient for picture fuzzy sets with the justification of its advantages. This correlation coefficient is better than the existing correlation coefficients and other such measures in the picture fuzzy theory because it considers the picture fuzzy set as a whole and also expresses the nature (positive or negative) as well as the extent of association between two PFSs. By performing some comparative analysis based on the computation of correlation degree and linguistic hedges, we establish the effectiveness of the suggested correlation measure over some available correlation measures in a picture fuzzy environment. Further, in the context of pattern recognition, we examine the performance of the proposed correlation measure over some existing picture fuzzy correlation measures. Finally, we apply the suggested picture fuzzy correlation coefficient to a decisionmaking problem involving the selection of an appropriate COVID-19 mask.
\end{abstract}

Keywords Picture fuzzy sets · Correlation coefficient · Pattern recognition · COVID-19

\section{Introduction}

Zadeh (1965) introduced the fuzzy set (FS) theory. After that, many authors Chen et al. (1995), Chen (1996), Chen and Jong (1997), Manoj et al. (1998), Chen and Huang (2003), Singh et al. (2019, 2020) have paid keen attention to FSs and their applications. In the FS theory, only the membership degree of an element is considered and the non-membership degree is automatically considered as one-minus membership degree. Therefore, one can certainly find out the non-membership degree of an element by knowing the membership degree of the same element.

Abdul Haseeb Ganie

ahg110605@gmail.com

Surender Singh

surender1976@gmail.com

1 Faculty of Sciences, School of Mathematics, Shri Mata

Vaishno Devi University, Katra,

Jammu and Kashmir 182320, India
However, in real-life situations, an experimenter may not be so certain about the non-membership degree due to the knowledge of membership degree (Deschrijver and Kerre 2003). In such a situation, an independent nonmembership function along with a membership function is desirable. Atanassov (1986) introduced the concept of intuitionistic fuzzy sets (IFSs) using membership and a non-membership function. For dealing with ambiguity and uncertainty, IFSs are more concrete and flexible than Zadeh's (1965) FSs in various circumstances. The concept of interval-valued IFSs (IVIFSs) was introduced by Atanassov and Gargov (1989) which is a generalization of IFSs in which the membership and non-membership degrees of an element are intervals. Some prominent applications of IFSs/IVIFSs can be found in clustering (Chen and Tan 1994; Huang 2012; Yue et al. 2013), decision-making (Chen and Tan 1994, Hong and Choi 2000; Chen and Chiou 2014; Chen and Huang 2017; Wang and Chen 2017; Zeng et al. 2019; Liu et al. 2020; Zeng et al. 2020; Zeng et al. 2020), medical diagnosis (De et al. 2001; Szmidt and 
Kacprzyk 2004; Zhang et al. 2012; Chen and Randyanto 2013), and pattern recognition (Zhang et al. 2012; Chen and Randyanto 2013; Chu et al. 2014). Although, IFSs have found a lot of applications in many areas still there are some situations in our day-to-day life that could not be handled by IFSs. For example, voting, medical diagnosis, feature selection, personal selection, etc. Therefore, to deal with such situations Cuong and Kreinovich (2013) introduced the notion of picture fuzzy set (PFS), which is a direct generalization of Zadeh's (1965) FSs, and Atanassov's (1986) IFSs. In a PFS, each element is assigned a membership degree, a non-membership degree, and a neutrality degree. Cuong (2014) defined some operations on PFSs, distance measure of PFSs, convex combination of PFSs, interval-valued PFSs (IVPFSs), PF relation, picture fuzzy soft set (PFSS), and applications of PFSs to a simple decision-making problem. Wei (2016) introduced PF crossentropy and applied it to multi-attribute decision-making (MADM). Wei (2017) introduced some PF cosine similarity measures and demonstrated their application in strategic decision-making. PF dice similarity measures with their application in building material recognition were introduced by Wei and Gao (2018). Wei (2018) introduced PF similarity measures and some PF weighted similarity measures and applied them to minerals field recognition and building material recognition. Some bi-parametric distance and similarity measures for PFSs with their application in medical diagnosis were introduced by Khan et al. (2020a). A new decision-making method in a generalized PFSS environment was proposed by Khan et al. (2020b). The present study is about the correlation coefficient for PFSs.

The main contributions of this study are described below:

- We suggest a new PF correlation measure that identifies both the type and degree of correlation between two PFSs.

- We demonstrate its use in pattern recognition and use "Degree of Confidence" to measure its performance.

- We use real data related to the Iris plant to test the proposed PF correlation measure. https://archive.ics. uci.edu/ml/datasets/Iris.

- We demonstrate its utility in MADM.

The remainder of the paper is structured as follows: In Sect. 2, we discuss the existing work related to the present study. Some basic definitions related to fuzzy/non-standard fuzzy theory together with the existing PF correlation measures are given in Sect. 3. Section 4 is devoted to the introduction of the novel PF correlation measure along with its properties. The comparative analysis of the proposed PF correlation measure with the existing PF correlation measures is also given in this section. The application of the proposed $\mathrm{PF}$ correlation measure in pattern recognition and also on the real data pertaining to the Iris plant (https://archive.ics.uci.edu/ml/datasets/Iris) is shown in Sect. 5. A novel application of the proposed PF correlation measure in MADM is also shown in this section. The conclusion and future scope of the present study are given in Sect. 6.

\section{Related work}

The correlation coefficient is a commonly used statistical tool and is an essential measure in decision-making, pattern recognition, classification, data analysis, and so on Bonizzoni et al. (2008), Cheung and Li (2012), Riegel et al. (2008), Kumar et al. (2011), Park et al. (2009), Wei et al. (2011). A correlation coefficient for Atanassov's (1986), Atanassov and Gargov (1989) IFSs was introduced by Gerstenkorn and Manko (1991). In probability spaces, Hong and Hwang (1995) introduced the notion of the correlation coefficient. By considering Atanassov's IFSs (1986) as a group of Zadeh's FSs (1965), Mitchell (2004) presented the correlation coefficient for Atanassov's (1986) IFSs. A method for calculating the correlation coefficient of Atanassov's (1986) IFSs with the help of the centroid method was developed by Hung and Wu (2002). Due to potential applications, Atanassov's (1986) IFSs correlation coefficient was extended to IVIFSs by Hong (1998) and Bustince and Burillo (1995). For hesitant fuzzy elements (HFEs), some correlation coefficients with their application in pattern recognition were introduced by $\mathrm{Xu}$ and $\mathrm{Xia}$ (2011). Later on, the notion of correlation coefficient was extended to interval-valued hesitant fuzzy sets (IVHFSs) by Chen et al. (2013) and introduced clustering algorithms by considering HFSs and IVHFSs. Recently, Singh and Lalotra (2019) introduced one and two parametric generalizations of the correlation coefficients introduced by $\mathrm{Xu}$ and Xia (2011) in the hesitant fuzzy environment and demonstrated their efficiency in clustering analysis. Singh and Lalotra (2018) and, Singh and Sharma (2019) investigated a generalization of correlation coefficients for fuzzy soft sets (FSSs) and hesitant fuzzy soft sets (HFSSs). In the PF environment, Singh (2015) introduced some correlation coefficients and demonstrated their effectiveness in bidirectional approximate reasoning besides presenting the $\mathrm{PF}$ clustering algorithm. Jin et al. (2019) introduced Pearson's picture fuzzy correlation coefficient with its application to decision-making. Recently, Ganie et al. (2020) introduced some new correlation coefficients in the PF environment and demonstrated their application in pattern recognition, medical diagnosis, and clustering. However, all these PF correlation coefficients neglect the integrity of PFSs. So, there is a need to construct a PF correlation coefficient that should consider the PFS as a whole and reflects the 
correlation more practically. Also, the existing PF correlation measures are not satisfactory from the viewpoint of the structured linguistic variables. Furthermore, all of the current PF correlation coefficients have some drawbacks, which are mentioned below.

- The PF correlation coefficients due to Singh (2015) only show the degree of correlation between two PFSs and reveal nothing about the type of correlation (positive or negative).

- The PF correlation coefficients proposed by Ganie et al. (2020) express both the type and extent of correlation between two PFSs, but they do not take into account the degree of refusal, leading to counter-intuitive results in most cases (Examples 1-5).

- Though the PF correlation coefficient introduced by Jin et al. (2019) takes into account all four membership degrees (membership, non-membership, neutrality, and refusal), it often produces erroneous results or fails to measure the correlation of the two PFSs (Examples 1$5)$.

- The Hung (2001) type PF correlation coefficient proposed by Ganie et al. (2020) fails to compute the correlation coefficient of two PFSs where each element's membership, non-membership, or neutrality degree is zero (Example 6).

- The PF correlation coefficient introduced by Jin et al. (2019) fails to quantify the correlation coefficient of the two PFSs in which each element's degree of refusal is zero (Examples 2, 5, and 7).

To address the above-mentioned limitations of the existing PF correlation measures, we in this paper introduce a novel PF correlation measure together with some properties.

\section{Preliminaries}

In this section, we give some basic definitions concerning the present study. Throughout this paper, let $Y=$ $\left\{y_{1}, y_{2}, \ldots, y_{n}\right\}$ be the universal set and $\operatorname{PFS}(Y)$ be the family of all PFSs on $Y$.

Definition 1 (Zadeh 1965) A fuzzy subset $R$ of the universal set $Y$ is defined as $R=\left\{\left(y_{i}, \theta_{R}\left(y_{i}\right)\right) \mid y_{i} \in Y\right\}$, where $\theta_{R}: Y \rightarrow[0,1]$ represents a membership function. The value $\theta_{R}\left(y_{i}\right)$ describes the extent of the presence of $y_{i} \in Y$ in $R$.

Definition 2 (Atanassov 1986) An IFS $R$ in $Y$ is defined as $R=\left\{\left(y_{i}, \theta_{R}\left(y_{i}\right), \tau_{R}\left(y_{i}\right)\right) \mid y_{i} \in Y\right\}$, where $\theta_{R}\left(y_{i}\right)$ and $\tau_{R}\left(y_{i}\right)$ represent the membership and non-membership degrees respectively of the element $y_{i} \in Y$ to the set $R$ with the conditions $\quad 0 \leq \theta_{R}\left(y_{i}\right), \tau_{R}\left(y_{i}\right) \leq 1$

and $0 \leq \theta_{R}\left(y_{i}\right)+\tau_{R}\left(y_{i}\right) \leq 1$.

Definition 3 (Atanassov 1986) Let the family of all IFSs over the universe of discourse $Y$ be $\operatorname{IFS}(Y)$. For any $R, S \in I F S(Y)$, some operations are defined as

- $R \subseteq S$ if and only if $\theta_{R}\left(y_{i}\right) \leq \theta_{S}\left(y_{i}\right)$ and $\tau_{R}\left(y_{i}\right) \geq \tau_{S}\left(y_{i}\right)$;

- $R=S$ if and only if $R \subseteq S$ and $R \supseteq S$;

- $R^{c}=\left\{\left(y_{i}, \tau_{R}\left(y_{i}\right), \theta_{R}\left(y_{i}\right)\right) \mid y_{i} \in Y\right\}$, where $c$ denotes the complement;

- $R \cup S=\left\{\left(y_{i}, \theta_{R}\left(y_{i}\right) \vee \theta_{S}\left(y_{i}\right), \tau_{R}\left(y_{i}\right) \wedge \quad \tau_{S}\left(y_{i}\right)\right) \mid y_{i} \in Y\right\}$, where " $\vee$ " denotes maximum and " $\wedge$ " denotes minimum;

- $R \cap S=\left\{\left(y_{i}, \theta_{R}\left(y_{i}\right) \wedge \theta_{S}\left(y_{i}\right), \tau_{R}\left(y_{i}\right) \vee \quad \tau_{S}\left(y_{i}\right)\right) \mid y_{i} \in Y\right\}$, where " $\vee$ " denotes maximum and " $\wedge$ " denotes minimum.

Definition 4 (Cuong and Kreinovich 2013) A PFS $R$ in $Y$ is defined as $R=\left\{\left(y_{i}, \theta_{R}\left(y_{i}\right), \delta_{R}\left(y_{i}\right), \tau_{R}\left(y_{i}\right)\right) \mid y_{i} \in Y\right\}$, where $\theta_{R}\left(y_{i}\right), \delta_{R}\left(y_{i}\right)$, and $\tau_{R}\left(y_{i}\right)$ represent the membership, the neutrality, and the non-membership degrees respectively of the element $y_{i} \in Y$ to set $R$ with the conditions $0 \leq \theta_{R}\left(y_{i}\right), \delta_{R}\left(y_{i}\right), \tau_{R}\left(y_{i}\right) \leq 1 \quad$ and $0 \leq \theta_{R}\left(y_{i}\right)+\delta_{R}\left(y_{i}\right)+\tau_{R}\left(y_{i}\right) \leq 1$.

Definition 5 (Cuong 2014): For any $R, S \in P F S(Y)$, some operations are given below.

- $R \subseteq S$ if and only if $\theta_{R}\left(y_{i}\right) \leq \theta_{S}\left(y_{i}\right), \delta_{R}\left(y_{i}\right) \leq \delta_{S}\left(y_{i}\right)$, and $\tau_{R}\left(y_{i}\right) \geq \tau_{S}\left(y_{i}\right)$

- $R=S$ if and only if $R \subseteq S$ and $R \supseteq S$;

- $R^{c}=\left\{\left(y_{i}, \tau_{R}\left(y_{i}\right), \delta_{R}\left(y_{i}\right), \theta_{R}\left(y_{i}\right)\right) \mid y_{i} \in Y\right\}$, where $c$ denotes the complement;

- $R \cup S=\left\{\left(y_{i}, \theta_{R}\left(y_{i}\right) \vee \theta_{S}\left(y_{i}\right), \delta_{R}\left(y_{i}\right) \wedge \delta_{S}\left(y_{i}\right), \tau_{R}\left(y_{i}\right)\right.\right.$ $\left.\left.\wedge \tau_{S}\left(y_{i}\right)\right) \mid y_{i} \in Y\right\}$, where " $\vee$ " denotes maximum and " $\wedge$ " denotes minimum;

- $R \cap S=\left\{\left(y_{i}, \theta_{R}\left(y_{i}\right) \wedge \theta_{S}\left(y_{i}\right), \delta_{R}\left(y_{i}\right) \wedge \delta_{S}\left(y_{i}\right), \tau_{R}\left(y_{i}\right)\right.\right.$ $\left.\left.\vee \tau_{S}\left(y_{i}\right)\right) \mid y_{i} \in Y\right\}$, where " $\vee$ " denotes maximum an " $\wedge$ " denotes minimum.

In the next section, we review the existing PF correlation measures.

\subsection{The existing PF correlation measures}

In this section, we revise the existing PF correlation measures available in the literature and also discuss their limitations.

Let $R, S \in P F S(Y)$.

PF correlation measures due to Singh (2015): 
$C_{S 1}(R, S)=\frac{\sum_{i=1}^{n}\left\{\theta_{R}\left(y_{i}\right) \theta_{S}\left(y_{i}\right)+\delta_{R}\left(y_{i}\right) \delta_{S}\left(y_{i}\right)+\tau_{R}\left(y_{i}\right) \tau_{S}\left(y_{i}\right)+\gamma_{R}\left(y_{i}\right) \gamma_{S}\left(y_{i}\right)\right\}}{\sqrt{\sum_{i=1}^{n}\left\{\left(\theta_{R}\left(y_{i}\right)\right)^{2}+\left(\delta_{R}\left(y_{i}\right)\right)^{2}+\left(\tau_{R}\left(y_{i}\right)\right)^{2}+\left(\gamma_{R}\left(y_{i}\right)\right)^{2}\right\} \times \sum_{i=1}^{n}\left\{\left(\theta_{S}\left(y_{i}\right)\right)^{2}+\left(\delta_{S}\left(y_{i}\right)\right)^{2}+\left(\tau_{S}\left(y_{i}\right)\right)^{2}+\left(\gamma_{S}\left(y_{i}\right)\right)^{2}\right\}}}$,

where $\gamma\left(y_{i}\right)=1-\left(\theta\left(y_{i}\right)+\delta\left(y_{i}\right)+\tau\left(y_{i}\right)\right)$ is the degree of refusal, and
The PF correlation coefficient $C_{\text {JWsZLP }}$ due to Jin et al. (2019) often gives the same correlation degree between different PFSs and also fails to compute the degree of

$C_{S 2}(R, S)=\frac{\sum_{i=1}^{n}\left\{\theta_{R}\left(y_{i}\right) \theta_{S}\left(y_{i}\right)+\delta_{R}\left(y_{i}\right) \delta_{S}\left(y_{i}\right)+\tau_{R}\left(y_{i}\right) \tau_{S}\left(y_{i}\right)+\gamma_{R}\left(y_{i}\right) \gamma_{S}\left(y_{i}\right)\right\}}{\max \left\{\sum_{i=1}^{n}\left\{\left(\theta_{R}\left(y_{i}\right)\right)^{2}+\left(\delta_{R}\left(y_{i}\right)\right)^{2}+\left(\tau_{R}\left(y_{i}\right)\right)^{2}+\left(\gamma_{R}\left(y_{i}\right)\right)^{2}\right\}, \sum_{i=1}^{n}\left\{\left(\theta_{S}\left(y_{i}\right)\right)^{2}+\left(\delta_{S}\left(y_{i}\right)\right)^{2}+\left(\tau_{S}\left(y_{i}\right)\right)^{2}+\left(\gamma_{S}\left(y_{i}\right)\right)^{2}\right\}\right\}}$.

The PF correlation coefficients $C_{S 1}$ and $C_{S 2}$ due to Singh (2015) give the degree of correlation between two PFSs but they fail to determine the nature of correlation.

PF correlation measure due to Jin et al. (2019):

$C_{\mathrm{JWSZLP}}(R, S)=\frac{1}{4}\left(\Psi_{1}(R, S)+\Psi_{2}(R, S)+\Psi_{3}(R, S)+\Psi_{4}(R, S)\right)$,

where,

$$
\begin{aligned}
\Psi_{1}(R, S) & =\frac{\sum_{i=1}^{n}\left(\theta_{R}\left(y_{i}\right)-\overline{\theta_{R}}\right)\left(\theta_{S}\left(y_{i}\right)-\overline{\theta_{S}}\right)}{\sqrt{\sum_{i=1}^{n}\left(\theta_{R}\left(y_{i}\right)-\overline{\theta_{R}}\right)^{2}} \sqrt{\sum_{i=1}^{n}\left(\theta_{S}\left(y_{i}\right)-\overline{\theta_{S}}\right)^{2}}}, \Psi_{2}(R, S)=\frac{\sum_{i=1}^{n}\left(\delta_{R}\left(y_{i}\right)-\overline{\delta_{R}}\right)\left(\delta_{S}\left(y_{i}\right)-\overline{\delta_{S}}\right)}{\sqrt{\sum_{i=1}^{n}\left(\delta_{R}\left(y_{i}\right)-\overline{\delta_{R}}\right)^{2}} \sqrt{\sum_{i=1}^{n}\left(\delta_{S}\left(y_{i}\right)-\overline{\delta_{S}}\right)^{2}}}, \\
\Psi_{3}(R, S) & =\frac{\sum_{i=1}^{n}\left(\tau_{R}\left(y_{i}\right)-\overline{\tau_{R}}\right)\left(\tau_{S}\left(y_{i}\right)-\overline{\tau_{S}}\right)}{\sqrt{\sum_{i=1}^{n}\left(\tau_{R}\left(y_{i}\right)-\overline{\tau_{R}}\right)^{2}} \sqrt{\sum_{i=1}^{n}\left(\tau_{S}\left(y_{i}\right)-\overline{\tau_{S}}\right)^{2}}}, \Psi_{4}(R, S)=\frac{\sum_{i=1}^{n}\left(\gamma_{R}\left(y_{i}\right)-\overline{\gamma_{R}}\right)\left(\gamma_{S}\left(y_{i}\right)-\overline{\gamma_{S}}\right)}{\sqrt{\sum_{i=1}^{n}\left(\gamma_{R}\left(y_{i}\right)-\overline{\gamma_{R}}\right)^{2}} \sqrt{\sum_{i=1}^{n}\left(\gamma_{S}\left(y_{i}\right)-\overline{\gamma_{S}}\right)^{2}}}, \\
\overline{\theta_{R}} & =\frac{1}{n} \sum_{i=1}^{n} \theta_{R}\left(y_{i}\right), \overline{\delta_{R}}=\frac{1}{n} \sum_{i=1}^{n} \delta_{R}\left(y_{i}\right), \overline{\tau_{R}}=\frac{1}{n} \sum_{i=1}^{n} \tau_{R}\left(y_{i}\right), \overline{\gamma_{R}}=\frac{1}{n} \sum_{i=1}^{n} \gamma_{R}\left(y_{i}\right), \overline{\theta_{S}}=\frac{1}{n} \sum_{i=1}^{n} \theta_{S}\left(y_{i}\right), \\
\overline{\delta_{S}} & =\frac{1}{n} \sum_{i=1}^{n} \delta_{S}\left(y_{i}\right), \overline{\tau_{S}}=\frac{1}{n} \sum_{i=1}^{n} \tau_{S}\left(y_{i}\right), \text { and } \overline{\gamma_{S}}=\frac{1}{n} \sum_{i=1}^{n} \gamma_{S}\left(y_{i}\right) .
\end{aligned}
$$

correlation between those PFSs in which the refusal degree of each element is zero.

PF correlation measures due to Ganie et al. (2020): where $\overline{\theta_{R}}, \overline{\theta_{S}}, \overline{\delta_{R}}, \overline{\delta_{S}}, \overline{\tau_{R}}, \overline{\tau_{S}}, \overline{\gamma_{R}}, \overline{\gamma_{S}}$ are the same as in Eq. (3) and, 


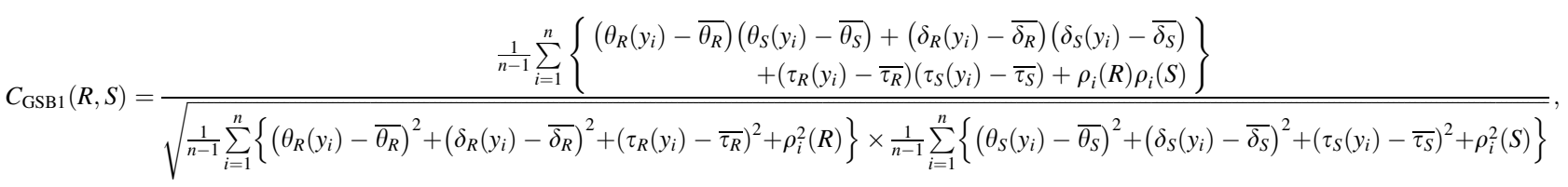

$$
\begin{aligned}
& \rho_{i}(R)=\left(\theta_{R}\left(y_{i}\right)-\overline{\theta_{R}}\right)-\left(\delta_{R}\left(y_{i}\right)-\overline{\delta_{R}}\right)-\left(\tau_{R}\left(y_{i}\right)-\overline{\tau_{R}}\right), \\
& \rho_{i}(S)=\left(\theta_{S}\left(y_{i}\right)-\overline{\theta_{S}}\right)-\left(\delta_{S}\left(y_{i}\right)-\overline{\delta_{S}}\right)-\left(\tau_{S}\left(y_{i}\right)-\overline{\tau_{S}}\right) \\
& \quad \forall i=1,2, \ldots, n . \\
& C_{\mathrm{GSB} 2}(R, S)=\frac{1}{3}\left(\Psi_{1}(R, S)+\Psi_{2}(R, S)+\Psi_{3}(R, S)\right),
\end{aligned}
$$

where, $\Psi_{i}(R, S), i=1,2,3$ are the same as in Eq. (3).

The PF correlation coefficients $C_{\mathrm{GSB} 1}$ and $C_{\mathrm{GSB} 2}$ due to Ganie et al. (2020) often gives same correlation degree between different PFSs and also the correlation coefficient $C_{\mathrm{GSB} 2}$ fails to compute the degree of correlation between those PFSs in which either the membership or non-membership or neutrality degree of each element is zero.

In the next section, we introduce a new correlation coefficient for PFSs.

\section{A novel correlation coefficient of PFSs}

In this section, we propose a novel correlation coefficient for PFSs which overcomes the limitations of the existing PF correlation coefficients.

Definition 6 Let $R, S \in P F S(Y)$, then the correlation coefficient between $R$ and $S$ is given by. where $\overline{\theta_{R}}=\frac{1}{n} \sum_{i=1}^{n} \theta_{R}\left(y_{i}\right), \overline{\delta_{R}}=\frac{1}{n} \sum_{i=1}^{n} \delta_{R}\left(y_{i}\right), \overline{\tau_{R}}=\frac{1}{n} \sum_{i=1}^{n} \tau_{R}\left(y_{i}\right)$, $\overline{\theta_{S}}=\frac{1}{n} \sum_{i=1}^{n} \theta_{S}\left(y_{i}\right), \overline{\delta_{S}}=\frac{1}{n} \sum_{i=1}^{n} \delta_{S}\left(y_{i}\right)$, and $\overline{\tau_{S}}=\frac{1}{n} \sum_{i=1}^{n} \tau_{S}\left(y_{i}\right)$.

The following Theorem 1 establishes some properties of the PF correlation coefficient $C_{\mathrm{GS}}(R, S)$.

Theorem 1 Let $R, S \in \operatorname{PFS}(Y)$, then.

(a) $C_{\mathrm{GS}}(R, S)=C_{\mathrm{GS}}(S, R)$;

(b) $C_{\mathrm{GS}}(R, S)=1$ if $R=S$;

(c) $\left|C_{\mathrm{GS}}(R, S)\right| \leq 1$;

(d) If $R=\alpha S$, then $C_{\mathrm{GS}}(R, S)=1$ for $\alpha>0$ and $C_{\mathrm{GS}}(R, S)=-1$ for $\alpha<0$.

Proof (a) We have

\section{.}




$$
\begin{aligned}
C_{\mathrm{GS}}(R, S) & =\frac{\sum_{i=1}^{n}\left\{\left(\left(\theta_{R}\left(y_{i}\right)-\overline{\theta_{R}}\right)+\left(\delta_{R}\left(y_{i}\right)-\overline{\delta_{R}}\right)+\left(\tau_{R}\left(y_{i}\right)-\overline{\tau_{R}}\right)\right) \times\left(\left(\theta_{S}\left(y_{i}\right)-\overline{\theta_{S}}\right)+\left(\delta_{S}\left(y_{i}\right)-\overline{\delta_{S}}\right)+\left(\tau_{S}\left(y_{i}\right)-\overline{\tau_{S}}\right)\right)\right\}}{\sqrt{\sum_{i=1}^{n}\left(\left(\theta_{R}\left(y_{i}\right)-\overline{\theta_{R}}\right)+\left(\delta_{R}\left(y_{i}\right)-\overline{\delta_{R}}\right)+\left(\tau_{R}\left(y_{i}\right)-\overline{\tau_{R}}\right)\right)^{2} \times \sum_{i=1}^{n}\left(\left(\theta_{S}\left(y_{i}\right)-\overline{\theta_{S}}\right)+\left(\delta_{S}\left(y_{i}\right)-\overline{\delta_{S}}\right)+\left(\tau_{S}\left(y_{i}\right)-\overline{\tau_{S}}\right)\right)^{2}}}, \\
& =\frac{\sum_{i=1}^{n}\left\{\left(\left(\theta_{S}\left(y_{i}\right)-\overline{\theta_{S}}\right)+\left(\delta_{S}\left(y_{i}\right)-\overline{\delta_{S}}\right)+\left(\tau_{S}\left(y_{i}\right)-\overline{\tau_{S}}\right)\right) \times\left(\left(\theta_{R}\left(y_{i}\right)-\overline{\theta_{R}}\right)+\left(\delta_{R}\left(y_{i}\right)-\overline{\delta_{R}}\right)+\left(\tau_{R}\left(y_{i}\right)-\overline{\tau_{R}}\right)\right)\right\}}{\sqrt{\sum_{i=1}^{n}\left(\left(\theta_{S}\left(y_{i}\right)-\overline{\theta_{S}}\right)+\left(\delta_{S}\left(y_{i}\right)-\overline{\delta_{S}}\right)+\left(\tau_{S}\left(y_{i}\right)-\overline{\tau_{S}}\right)\right)^{2} \times \sum_{i=1}^{n}\left(\left(\theta_{R}\left(y_{i}\right)-\overline{\theta_{R}}\right)+\left(\delta_{R}\left(y_{i}\right)-\overline{\delta_{R}}\right)+\left(\tau_{R}\left(y_{i}\right)-\overline{\tau_{R}}\right)\right)^{2}}} \\
& =C_{\mathrm{GS}}(S, R) .
\end{aligned}
$$

(b) Let $R=S$, i.e., $\theta_{R}\left(y_{i}\right)=\theta_{S}\left(y_{i}\right), \delta_{R}\left(y_{i}\right)=\delta_{S}\left(y_{i}\right)$, and $\tau_{R}\left(y_{i}\right)=\tau_{S}\left(y_{i}\right), \forall i=1,2, \ldots, n$. Then

$$
\begin{aligned}
C_{\mathrm{GS}}(R, S) & =\frac{\sum_{i=1}^{n}\left\{\left(\left(\theta_{R}\left(y_{i}\right)-\overline{\theta_{R}}\right)+\left(\delta_{R}\left(y_{i}\right)-\overline{\delta_{R}}\right)+\left(\tau_{R}\left(y_{i}\right)-\overline{\tau_{R}}\right)\right) \times\left(\left(\theta_{S}\left(y_{i}\right)-\overline{\theta_{S}}\right)+\left(\delta_{S}\left(y_{i}\right)-\overline{\delta_{S}}\right)+\left(\tau_{S}\left(y_{i}\right)-\overline{\tau_{S}}\right)\right)\right\}}{\sqrt{\sum_{i=1}^{n}\left(\left(\theta_{R}\left(y_{i}\right)-\overline{\theta_{R}}\right)+\left(\delta_{R}\left(y_{i}\right)-\overline{\delta_{R}}\right)+\left(\tau_{R}\left(y_{i}\right)-\overline{\tau_{R}}\right)\right)^{2} \times \sum_{i=1}^{n}\left(\left(\theta_{S}\left(y_{i}\right)-\overline{\theta_{S}}\right)+\left(\delta_{S}\left(y_{i}\right)-\overline{\delta_{S}}\right)+\left(\tau_{S}\left(y_{i}\right)-\overline{\tau_{S}}\right)\right)^{2}}}, \\
& =\frac{\sum_{i=1}^{n}\left(\left(\theta_{S}\left(y_{i}\right)-\overline{\theta_{S}}\right)+\left(\delta_{S}\left(y_{i}\right)-\overline{\delta_{S}}\right)+\left(\tau_{S}\left(y_{i}\right)-\overline{\tau_{S}}\right)\right)^{2}}{\sqrt{\left(\sum_{i=1}^{n}\left(\left(\theta_{S}\left(y_{i}\right)-\overline{\theta_{S}}\right)+\left(\delta_{S}\left(y_{i}\right)-\overline{\delta_{S}}\right)+\left(\tau_{S}\left(y_{i}\right)-\overline{\tau_{S}}\right)\right)^{2}\right)^{2}}}=1 .
\end{aligned}
$$

(c) Using Cauchy-Schwarz inequality, we have

$$
\begin{aligned}
\left(C_{\mathrm{GS}}(R, S)\right)^{2} & =\left(\frac{\sum_{i=1}^{n}\left\{\left(\left(\theta_{R}\left(y_{i}\right)-\overline{\theta_{R}}\right)+\left(\delta_{R}\left(y_{i}\right)-\overline{\delta_{R}}\right)+\left(\tau_{R}\left(y_{i}\right)-\overline{\tau_{R}}\right)\right) \times\left(\left(\theta_{S}\left(y_{i}\right)-\overline{\theta_{S}}\right)+\left(\delta_{S}\left(y_{i}\right)-\overline{\delta_{S}}\right)+\left(\tau_{S}\left(y_{i}\right)-\overline{\tau_{S}}\right)\right)\right\}}{\sqrt{\sum_{i=1}^{n}\left(\left(\theta_{R}\left(y_{i}\right)-\overline{\theta_{R}}\right)+\left(\delta_{R}\left(y_{i}\right)-\overline{\delta_{R}}\right)+\left(\tau_{R}\left(y_{i}\right)-\overline{\tau_{R}}\right)\right)^{2} \times \sum_{i=1}^{n}\left(\left(\theta_{S}\left(y_{i}\right)-\overline{\theta_{S}}\right)+\left(\delta_{S}\left(y_{i}\right)-\overline{\delta_{S}}\right)+\left(\tau_{S}\left(y_{i}\right)-\overline{\tau_{S}}\right)\right)^{2}}}\right)^{2} \\
& \leq \frac{\sum_{i=1}^{n}\left(\left(\theta_{R}\left(y_{i}\right)-\overline{\theta_{R}}\right)+\left(\delta_{R}\left(y_{i}\right)-\overline{\delta_{R}}\right)+\left(\tau_{R}\left(y_{i}\right)-\overline{\tau_{R}}\right)\right)^{2} \times \sum_{i=1}^{n}\left(\left(\theta_{S}\left(y_{i}\right)-\overline{\theta_{S}}\right)+\left(\delta_{S}\left(y_{i}\right)-\overline{\delta_{S}}\right)+\left(\tau_{S}\left(y_{i}\right)-\overline{\tau_{S}}\right)\right)^{2}}{\left(\sqrt{\sum_{i=1}^{n}\left(\left(\theta_{R}\left(y_{i}\right)-\overline{\theta_{R}}\right)+\left(\delta_{R}\left(y_{i}\right)-\overline{\delta_{R}}\right)+\left(\tau_{R}\left(y_{i}\right)-\overline{\tau_{R}}\right)\right)^{2} \times \sum_{i=1}^{n}\left(\left(\theta_{S}\left(y_{i}\right)-\overline{\theta_{S}}\right)+\left(\delta_{S}\left(y_{i}\right)-\overline{\delta_{S}}\right)+\left(\tau_{S}\left(y_{i}\right)-\overline{\tau_{S}}\right)\right)^{2}}\right)^{2}}=1,
\end{aligned}
$$

or $\left|C_{G S}(R, S)\right| \leq 1$.

(d) Let $R=\alpha S$, then we have 


$$
\begin{aligned}
& C_{\mathrm{GS}}(R, S)=\frac{\sum_{i=1}^{n}\left\{\left(\left(\theta_{R}\left(y_{i}\right)-\overline{\theta_{R}}\right)+\left(\delta_{R}\left(y_{i}\right)-\overline{\delta_{R}}\right)+\left(\tau_{R}\left(y_{i}\right)-\overline{\tau_{R}}\right)\right) \times\left(\left(\theta_{S}\left(y_{i}\right)-\overline{\theta_{S}}\right)+\left(\delta_{S}\left(y_{i}\right)-\overline{\delta_{S}}\right)+\left(\tau_{S}\left(y_{i}\right)-\overline{\tau_{S}}\right)\right)\right\}}{\sqrt{\sum_{i=1}^{n}\left(\left(\theta_{R}\left(y_{i}\right)-\overline{\theta_{R}}\right)+\left(\delta_{R}\left(y_{i}\right)-\overline{\delta_{R}}\right)+\left(\tau_{R}\left(y_{i}\right)-\overline{\tau_{R}}\right)\right)^{2} \times \sum_{i=1}^{n}\left(\left(\theta_{S}\left(y_{i}\right)-\overline{\theta_{S}}\right)+\left(\delta_{S}\left(y_{i}\right)-\overline{\delta_{S}}\right)+\left(\tau_{S}\left(y_{i}\right)-\overline{\tau_{S}}\right)\right)^{2}}}, \\
& =\frac{\sum_{i=1}^{n}\left\{\left(\left(\alpha \theta_{S}\left(y_{i}\right)-\alpha \overline{\theta_{S}}\right)+\left(\alpha \delta_{S}\left(y_{i}\right)-\alpha \overline{\delta_{S}}\right)+\left(\alpha \tau_{S}\left(y_{i}\right)-\alpha \overline{\tau_{S}}\right)\right) \times\left(\left(\theta_{S}\left(y_{i}\right)-\overline{\theta_{S}}\right)+\left(\delta_{S}\left(y_{i}\right)-\overline{\delta_{S}}\right)+\left(\tau_{S}\left(y_{i}\right)-\overline{\tau_{S}}\right)\right)\right\}}{\sqrt{\sum_{i=1}^{n}\left(\left(\alpha \theta_{S}\left(y_{i}\right)-\alpha \overline{\theta_{S}}\right)+\left(\alpha \delta_{S}\left(y_{i}\right)-\alpha \overline{\delta_{S}}\right)+\left(\alpha \tau_{S}\left(y_{i}\right)-\alpha \overline{\tau_{S}}\right)\right)^{2} \times \sum_{i=1}^{n}\left(\left(\theta_{S}\left(y_{i}\right)-\overline{\theta_{S}}\right)+\left(\delta_{S}\left(y_{i}\right)-\overline{\delta_{S}}\right)+\left(\tau_{S}\left(y_{i}\right)-\overline{\tau_{S}}\right)\right)^{2}}}, \\
& =\frac{\alpha \sum_{i=1}^{n}\left(\left(\theta_{S}\left(y_{i}\right)-\overline{\theta_{S}}\right)+\left(\delta_{S}\left(y_{i}\right)-\overline{\delta_{S}}\right)+\left(\tau_{S}\left(y_{i}\right)-\overline{\tau_{S}}\right)\right)^{2}}{\sqrt{\alpha^{2}\left(\sum_{i=1}^{n}\left(\left(\theta_{S}\left(y_{i}\right)-\overline{\theta_{S}}\right)+\left(\delta_{S}\left(y_{i}\right)-\overline{\delta_{S}}\right)+\left(\tau_{S}\left(y_{i}\right)-\overline{\tau_{S}}\right)\right)^{2}\right)^{2}}}=\left\{\begin{array}{c}
-1, \text { if } \alpha<0 \\
1, \text { if } \alpha>0
\end{array} .\right.
\end{aligned}
$$

\subsection{Comparative analysis}

In this section, we perform some comparative analysis of the proposed PF correlation coefficient with some existing $\mathrm{PF}$ correlation coefficients through many aspects such as computation of correlation between different PFSs and linguistic hedges. First, we perform the comparative analysis based on correlation degree computation.

\subsubsection{Computation of correlation between different PFSs}

We solve some examples related to the calculation of correlation between different PFSs with the help of various PF correlation coefficients.

Example 1 Let $R, S, T \in P F S(Y)$ be given as $R=$ $\left\{\left(y_{1}, 0.64,0.112,0.13\right),\left(y_{2}, 0.4,0.5,0.1\right)\right\}, \quad S=\left\{\left(y_{1}, 0.5\right.\right.$, $\left.0.29,0.056),\left(y_{2}, 0.41,0.2,0.2\right)\right\} \quad$ and $T=\left\{\left(y_{1}, 0.1,0.3\right.\right.$, $\left.0.5),\left(y_{2}, 0.6,0.2,0.1\right)\right\}$. Clearly, $R \neq S$ but we have

$$
\begin{aligned}
C_{\mathrm{GSB} 1}(R, T) & =C_{\mathrm{GSB} 1}(S, T)=-0.8303, \\
C_{\mathrm{GSB} 2}(R, T) & =C_{\mathrm{GSB} 2}(S, T)=-0.3333, \\
C_{\mathrm{JWSZLP}}(R, T) & =C_{\mathrm{JWSZLP}}(S, T)=\text { Null, } \\
C_{\mathrm{GS}}(R, T) & =0, C_{\mathrm{GS}}(S, T)=0.1542 .
\end{aligned}
$$

Thus, only our proposed PF correlation measure differentiates between $R$ and $S$.

Example 2 Let $R, S, T \in P F S(Y)$ be given as $R=\left\{\left(y_{1}, 0.5,0.5,0.0\right),\left(y_{2}, 0.4,0.6,0.0\right)\right\}, \quad S=\left\{\left(y_{1}\right.\right.$, $\left.1.0,0.0,0.0),\left(y_{2}, 0.7,0.3,0.0\right)\right\} \quad$ and $T=\left\{\left(y_{1}, 0.8\right.\right.$, $\left.0.1,0.1),\left(y_{2}, 0.9,0.1,0.0\right)\right\}$. Clearly, $R \neq S$ but we have

$$
\begin{aligned}
C_{\mathrm{GSB} 1}(R, T) & =C_{\mathrm{GSB} 1}(S, T)=-0.8333, \\
C_{\mathrm{GSB} 2}(R, T) & =C_{\mathrm{GSB} 2}(S, T)=\text { Null }, \\
C_{\mathrm{JWSZLP}}(R, T) & =C_{\mathrm{JWSZLP}}(S, T)=\mathrm{Null}, \\
C_{\mathrm{GS}}(R, T) & =0.9701, C_{\mathrm{GS}}(S, T)=0.2425 .
\end{aligned}
$$

Here, we observe that the degree of refusal of each element in the PFSs $R, S$, and $T$ is zero and also $R \neq S$. The $\mathrm{PF}$ correlation coefficients $C_{\mathrm{GSB} 2}$ and $C_{\mathrm{JWSZLP}}$ fails to calculate the degree of correlation, and also the PF correlation coefficient $C_{\mathrm{GSB} 1}$ gives the same degree of correlation of the two different PFSs $R$ and $S$ with the PFS $T$. Our proposed $\mathrm{PF}$ correlation coefficient $C_{\mathrm{GS}}$ gives quite good results.

Example 3 Let $R, S, T \in P F S(Y)$ be given as $R=\left\{\left(y_{1}, 0.2,0.2,0.2\right),\left(y_{2}, 0.1,0.1,0.1\right)\right\}, \quad S=\left\{\left(y_{1}, 0.3\right.\right.$, $\left.0.3,0.3),\left(y_{2}, 0.2,0.2,0.2\right)\right\} \quad$ and $\quad T=\left\{\left(y_{1}, 0.4,0.3\right.\right.$, $\left.0.2),\left(y_{2}, 0.5,0.0,0.4\right)\right\}$. Clearly, $R \neq S$ but we have

$$
\begin{aligned}
C_{\mathrm{GSB} 1}(R, T) & =C_{\mathrm{GSB} 1}(S, T)=0.2357, \\
C_{\mathrm{GSB} 2}(R, T) & =C_{\mathrm{GSB} 2}(S, T)=-0.3333, \\
C_{\mathrm{JWSZLP}}(R, T) & =C_{\mathrm{JWSZLP}}(S, T)=-0.5000, \\
C_{\mathrm{GS}}(R, T) & =0.2776, C_{\mathrm{GS}}(S, T)=0.0000 .
\end{aligned}
$$

Here, we also observe that only our proposed correlation measure gives satisfactory results.

Example 4 Let $R, S, T \in P F S(Y)$ be given as $R=\left\{\left(y_{1}, 0.0,0.7,0.3\right),\left(y_{2}, 0.5,0.11,0.16\right)\right\}, \quad S=\left\{\left(y_{1}\right.\right.$, $\left.0.4,0.3,0.3),\left(y_{2}, 0.7,0.1,0.2\right)\right\} \quad$ and $T=\left\{\left(y_{1}, 0.8\right.\right.$, $\left.0.1,0.1),\left(y_{2}, 0.2,0.6,0.2\right)\right\}$. Here, $R \neq S$ but we have 


$$
\begin{aligned}
C_{\mathrm{GSB} 1}(R, T) & =C_{\mathrm{GSB} 1}(S, T)=-0.9952, \\
C_{\mathrm{GSB} 2}(R, T) & =C_{\mathrm{GSB} 2}(S, T)=-1.0000, \\
C_{\mathrm{JWSZLP}}(R, T) & =C_{\mathrm{JWSZLP}}(S, T)=\text { Null, } \\
C_{\mathrm{GS}}(R, T) & =0.7071, C_{\mathrm{GS}}(S, T)=-0.5547 .
\end{aligned}
$$

Thus, the performance of our proposed correlation measure $C_{\mathrm{GS}}$ is more reasonable than the existing PF correlation measures.

Example 5 Let $R, S, T \in P F S(Y)$ be given as $R=\left\{\left(y_{1}, 0.9,0.0,0.1\right),\left(y_{2}, 0.6,0.2,0.2\right)\right\}, \quad S=\left\{\left(y_{1}, 0.5\right.\right.$, $\left.0.2,0.3),\left(y_{2}, 0.2,0.4,0.4\right)\right\} \quad$ and $\quad T=\left\{\left(y_{1}, 0.5,0.1\right.\right.$, $\left.0.4),\left(y_{2}, 0.3,0.3,0.4\right)\right\}$. Clearly, $R \neq S$ but we have

$$
\begin{aligned}
C_{\mathrm{GSB} 1}(R, T) & =C_{\mathrm{GSB} 1}(S, T)=0.9815, \\
C_{\mathrm{GSB} 2}(R, T) & =C_{\mathrm{GSB} 2}(S, T)=\text { Null, } \\
C_{\mathrm{JWSZLP}}(R, T) & =C_{\mathrm{JWSZLP}}(S, T)=\mathrm{Null}, \\
C_{\mathrm{GS}}(R, T) & =0.8944, C_{\mathrm{GS}}(S, T)=-0.8944 .
\end{aligned}
$$

Here, our proposed PF correlation measure $C_{\mathrm{GS}}$ gives reasonable results, and the existing $\mathrm{PF}$ correlation measures $C_{\mathrm{GSB} 1}, C_{\mathrm{GSB} 2}$, and $C_{\mathrm{JWSZLP}}$ give unreasonable results.

Thus, from Examples 1-5, we observe that the degree of correlation of a PFS $T$ with two different PFSs $R$ and $S$ comes out to be the same by all of the existing PF correlation measures. This indicates that the existing PF correlation coefficients fail to distinguish the two different PFSs $R$ and $S$ and considers them to be identical, which is not reasonable. Also, in Examples 1, 2, 4, and 5, we see that the PF correlation coefficients $C_{\mathrm{GSB} 2}$ and $C_{\mathrm{JWSZLP}}$ fail to compute the correlation degree of the PFS $T$ with the two PFSs $R$ and $S$. However, in all these Examples, our proposed $\mathrm{PF}$ correlation coefficient $C_{\mathrm{GS}}$ performs very well without any unreasonable results. This implies that the proposed $\mathrm{PF}$ correlation coefficient $C_{\mathrm{GS}}$ is very effective than the existing PF correlation coefficients in computing correlation degrees between different PFSs.

Next in Examples 6 and 7, we show that the PF correlation coefficients $C_{\mathrm{GSB} 2}$ (Ganie et al. 2020) and $C_{J W S Z L P}$ (Jin et al. 2019) fail to compute the correlation degree of those PFSs in which either of the membership grades is zero.

Example 6 Let $R, S, T \in \operatorname{PFS}(Y)$ be such that the membership or non-membership or the neutrality degree of each element is zero. We show that the PF correlation measure $C_{\mathrm{GSB} 2}$ given in Eq. (5) is unable to calculate the correlation between them.

Case I When the degree of membership of each element in each of the PFSs $R, S$ and $T$ is zero i.e.,
$\theta_{R}\left(y_{i}\right)=\theta_{S}\left(y_{i}\right)=\theta_{T}\left(y_{i}\right)=0 \forall i=1,2, \ldots, n$, then the value of the factor $\Psi_{1}$ in the expression of $C_{G S B 2}$ cannot be calculated and therefore $C_{G S B 2}$ fails to calculate the degree of correlation between the PFSs $R, S$, and $T$.

\begin{tabular}{|c|c|c|c|c|c|}
\hline & M.L.L. & L. & V.L. & V.V.L. & N.V.L. \\
\hline \multicolumn{6}{|l|}{$C_{S 1}$} \\
\hline M.L.L. & 1.0000 & 0.8700 & 0.6102 & 0.4430 & 0.5887 \\
\hline L. & 0.8700 & 1.0000 & 0.9060 & 0.7596 & 0.7505 \\
\hline V.L. & 0.6102 & 0.9060 & 1.0000 & 0.9494 & 0.6625 \\
\hline V.V.L. & 0.4430 & 0.7596 & 0.9494 & 1.0000 & 0.5192 \\
\hline N.V.L. & 0.5887 & 0.7505 & 0.6625 & 0.5192 & 1.0000 \\
\hline \multicolumn{6}{|l|}{$C_{S 2}$} \\
\hline M.L.L. & 1.0000 & 0.8371 & 0.5423 & 0.3318 & 0.5233 \\
\hline L. & 0.8371 & 1.0000 & 0.7748 & 0.5475 & 0.6418 \\
\hline V.L. & 0.5423 & 0.7748 & 1.0000 & 0.8001 & 0.6625 \\
\hline V.V.L. & 0.3318 & 0.5475 & 0.8001 & 1.0000 & 0.4375 \\
\hline N.V.L. & 0.5233 & 0.6418 & 0.6625 & 0.4375 & 1.0000 \\
\hline \multicolumn{6}{|l|}{$C_{\text {JWSZLP }}$} \\
\hline M.L.L. & 1.0000 & 0.9705 & 0.8842 & 0.8003 & 0.4143 \\
\hline L. & 0.9705 & 1.0000 & 0.9679 & 0.9080 & 0.3463 \\
\hline V.L. & 0.8842 & 0.9679 & 1.0000 & 0.9794 & 0.2998 \\
\hline V.V.L. & 0.8003 & 0.9080 & 0.9794 & 1.0000 & 0.2899 \\
\hline N.V.L. & 0.4143 & 0.3463 & 0.2998 & 0.2899 & 1.0000 \\
\hline \multicolumn{6}{|l|}{$C_{\mathrm{GSB} 1}$} \\
\hline M.L.L. & 1.0000 & 0.8879 & 0.5872 & 0.4597 & -0.0764 \\
\hline L. & 0.8879 & 1.0000 & 0.8930 & 0.8106 & -0.4469 \\
\hline V.L. & 0.5872 & 0.8930 & 1.0000 & 0.9836 & -0.7011 \\
\hline V.V.L. & 0.4597 & 0.8106 & 0.9836 & 1.0000 & -0.7337 \\
\hline N.V.L. & -0.0764 & -0.4469 & -0.7011 & -0.7337 & 1.0000 \\
\hline \multicolumn{6}{|l|}{$C_{\mathrm{GSB} 2}$} \\
\hline M.L.L. & 1.0000 & 0.9633 & 0.8640 & 0.7899 & 0.2374 \\
\hline $\mathrm{L}$. & 0.9633 & 1.0000 & 0.9644 & 0.9129 & 0.1356 \\
\hline V.L. & 0.8640 & 0.9644 & 1.0000 & 0.9838 & 0.0664 \\
\hline V.V.L. & 0.7899 & 0.9129 & 0.9838 & 1.0000 & 0.0645 \\
\hline N.V.L. & 0.2374 & 0.1356 & 0.0664 & 0.0645 & 1.0000 \\
\hline \multicolumn{6}{|l|}{$C_{\mathrm{GS}}$} \\
\hline M.L.L. & 1.0000 & 0.9922 & 0.9450 & 0.8313 & 0.9450 \\
\hline L. & 0.9922 & 1.0000 & 0.9784 & 0.8933 & 0.9784 \\
\hline V.L. & 0.9450 & 0.9784 & 1.0000 & 0.9663 & 1.0000 \\
\hline V.V.L. & 0.8313 & 0.8933 & 0.9663 & 1.0000 & 0.9663 \\
\hline N.V.L. & 0.9450 & 0.9784 & 1.0000 & 0.9663 & 1.0000 \\
\hline
\end{tabular}

Case II When the degree of neutrality of each element in each of the PFSs $R, S$ and $T$ is zero i.e., $\delta_{R}\left(y_{i}\right)=\delta_{S}\left(y_{i}\right)=\delta_{T}\left(y_{i}\right)=0 \forall i=1,2, \ldots, n$, then the value of the factor $\Psi_{2}$ in the expression of $C_{\mathrm{GSB} 2}$ cannot be calculated and therefore $C_{\mathrm{GSB} 2}$ fails to calculate the degree of correlation between the PFSs $R, S$, and $T$.

Table 1 Computed correlation values regarding Example 8 
Table 2 Calculated values of various $\mathrm{PF}$-correlation measures regarding Example 9
Table 3 Calculated values of various $\mathrm{PF}$-correlation measures regarding Example 10

\begin{tabular}{llllll}
\hline & $\left(V_{1}, \mathrm{~W}\right)$ & $\left(V_{2}, \mathrm{~W}\right)$ & $\left(V_{3}, \mathrm{~W}\right)$ & Result & DoC \\
\hline$C_{S 1}$ (Singh 2015) & 0.9120 & 0.9150 & 0.4800 & $V_{2}$ & 0.4380 \\
$C_{S 2}$ (Singh 2015) & 0.8723 & 0.8571 & 0.3476 & $V_{1}$ & 0.5400 \\
$C_{\text {JWSZLP }}($ Jin et al. 2019) & Null & Null & Null & Not Classified & Null \\
$C_{\mathrm{GSB} 1}$ (Ganie et al. 2020) & -0.6325 & -0.4564 & -0.3450 & $V_{3}$ & 0.3989 \\
$C_{\mathrm{GSB} 2}$ (Ganie et al. 2020) & Null & Null & Null & Not Classified & Null \\
$C_{\mathrm{GS}}$ (Proposed) & 1.0000 & -0.8660 & -1.0000 & $V_{1}$ & 3.8660 \\
\hline
\end{tabular}

"Null" means cannot be calculated

\begin{tabular}{lrrrrr}
\hline & \multicolumn{1}{c}{$\left(V_{1}, \mathrm{~W}\right)$} & \multicolumn{1}{c}{$\left(V_{2}, \mathrm{~W}\right)$} & $\left(V_{3}, \mathrm{~W}\right)$ & Result & DoC \\
\hline$C_{S 1}($ Singh 2015) & 0.6885 & 0.7069 & 0.7341 & $V_{3}$ & 0.0728 \\
$C_{S 2}$ (Singh 2015) & 0.5926 & 0.6235 & 0.6049 & $V_{2}$ & 0.0494 \\
$C_{\text {JWSZLP }}($ Jin et al. 2019) & -0.3432 & -0.0968 & -0.1934 & $V_{2}$ & 0.3429 \\
$C_{\mathrm{GSB} 1}($ Ganie et al. 2020) & 0.2400 & -0.1096 & 0.1702 & $V_{1}$ & 0.4194 \\
$C_{\mathrm{GSB} 2}$ (Ganie et al. 2020) & -0.1689 & -0.2958 & -0.0911 & $V_{3}$ & 0.2824 \\
$C_{\mathrm{GS}}$ (Proposed) & -0.8660 & 0.5000 & -0.5000 & $V_{2}$ & 2.3660 \\
\hline
\end{tabular}

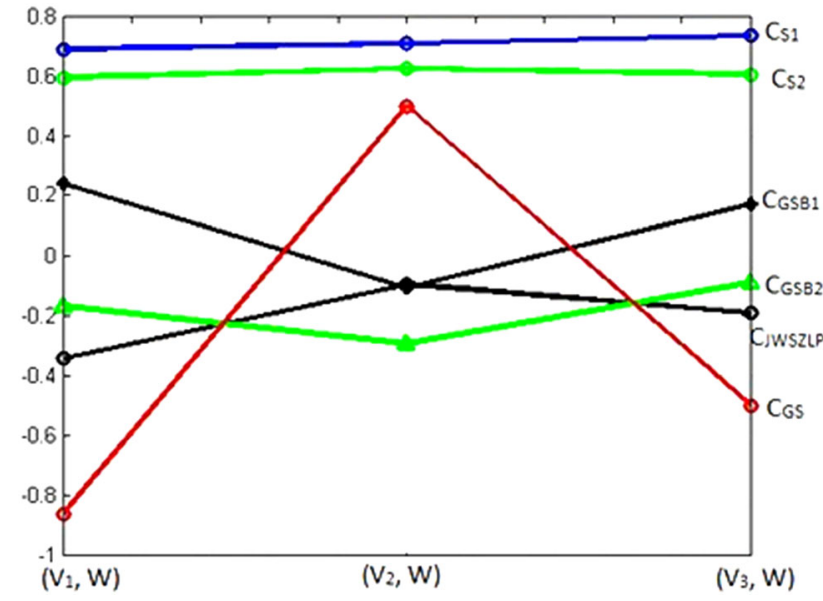

Fig. 1 Correlation coefficient of the unknown pattern with the known patterns

Case III When the degree of non-membership of each element in each of the PFSs $R, S$, and $T$ is zero i.e., $\tau_{R}\left(y_{i}\right)=\tau_{S}\left(y_{i}\right)=\tau_{T}\left(y_{i}\right)=0 \forall i=1,2, \ldots, n$, then the value of the factor $\Psi_{3}$ in the expression of $C_{\mathrm{GSB} 2}$ can not be calculated and therefore $C_{\mathrm{GSB} 2}$ fails to calculate the degree of correlation between the PFSs $R, S$, and $T$.

Example 7 Let $R, S, T \in \operatorname{PFS}(Y)$ be such that the refusal degree of each element is zero, then the value of the factor $\Psi_{4}$ in the expression of $C_{\text {JWSZLP }}$ cannot be calculated and

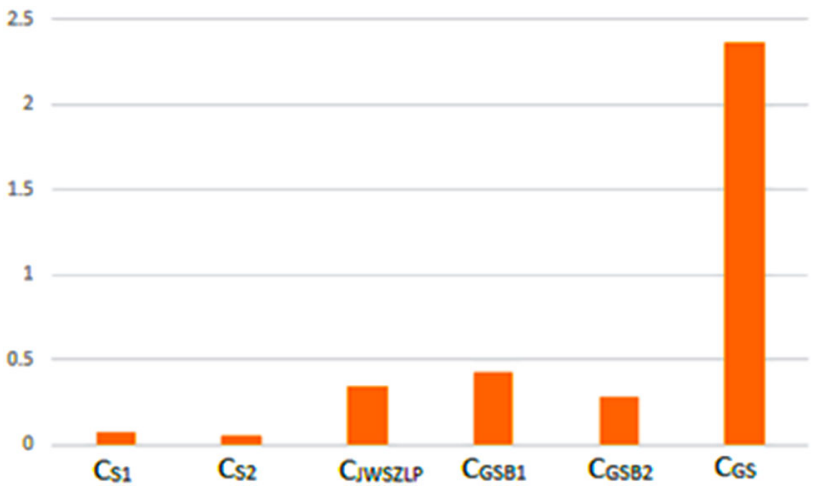

Fig. 2 Degree of Confidence of various PF correlation coefficients

therefore $C_{\text {JWSZLP }}$ fails to calculate the degree of correlation between the PFSs $R, S$, and $T$.

\subsubsection{Linguistic hedges}

The structured linguistic investigation of all ambiguity/vagueness measures in standard and non-standard versions offers clear theoretical proof of a measure's applicability in realistic situations. Zadeh (1972) was the first to propose the idea of standardized linguistic variables or linguistic hedges in the context of FSs. De et al. (2000) later expanded the definition to IFSs. The modifier $R^{\alpha}$ of a 
Table 4 Calculated values of various $\mathrm{PF}$-correlation measures regarding Example 11

\begin{tabular}{llllll}
\hline & $\left(V_{1}, \mathrm{~W}\right)$ & $\left(V_{2}, \mathrm{~W}\right)$ & $\left(V_{3}, \mathrm{~W}\right)$ & Result & DoC \\
\hline$C_{S 1}($ Singh 2015) & $\mathbf{0 . 9 8 4 6}$ & $\mathbf{0 . 9 8 4 6}$ & 0.9749 & Not Classified & Null \\
$C_{S 2}$ (Singh 2015) & $\mathbf{0 . 9 7 4 5}$ & $\mathbf{0 . 9 7 4 5}$ & 0.9505 & Not Classified & Null \\
$C_{\mathrm{JWSZLP}}($ Jin et al. 2019) & Null & Null & Null & Not Classified & Null \\
$C_{\mathrm{GSB} 1}($ Ganie et al. 2020) & 0.8896 & 0.9511 & 0.9785 & $V_{3}$ & 0.0528 \\
$C_{\mathrm{GSB} 2}$ (Ganie et al. 2020) & Null & Null & Null & Not Classified & Null \\
$C_{\mathrm{GS}}$ (Proposed) & 0.8000 & 0.9467 & 0.3162 & $V_{2}$ & 0.7772 \\
\hline
\end{tabular}

Bold values indicate unreasonable results. "Null" means cannot be calculated
PFS $R$, where $\alpha$ is a positive real number was given by Wang et al. (2017) and is defined as:

$R^{\alpha}=\left\{y_{i},\left(\theta_{R}\left(y_{i}\right)+\delta_{R}\left(y_{i}\right)\right)^{\alpha}-\left(\delta_{R}\left(y_{i}\right)\right)^{\alpha},\left(\delta_{R}\left(y_{i}\right)\right)^{\alpha}\right.$, $\left.1-\left(1-\tau_{R}\left(y_{i}\right)\right)^{\alpha} \mid y_{i} \in Y\right\}$.

With the help of Eq. (7), Singh and Ganie (2021) defined various linguistic hedges such as LARGE (L.), very LARGE (V.L.), very very LARGE (V.V.L.), not very LARGE (N.V.L.), and more or less LARGE (M.L.L.) in the PF environment. We now apply various PF correlation measures for computing the correlation between the linguistic hedges L., V.L., V.V.L., N.V.L., and M.L.L. in the example given below.

Example 8 (Singh and Ganie 2021) Consider a PFS $R$ in the domain $Y$ given as.

$R=$

$\left\{\begin{array}{l}\left(y_{1}, 0.20,0.15,0.40\right),\left(y_{2}, 0.35,0.11,0.54\right),\left(y_{3}, 0.32,0.19,0.12\right), \\ \left(y_{4}, 0.49,0.31,0.20\right),\left(y_{5}, 0.16,0.36,0.25\right)\end{array}\right\}$.

With the help of the modifier of a PFS $R$ given in the Eq. (7), the PFSs $R^{\frac{1}{2}}, R^{2}, R^{4}$, and $\left(R^{2}\right)^{c}$ can be determined. Due to the characterization of linguistic hedges, we regard $R$ as 'LARGE', $R^{\frac{1}{2}}$ as 'more or less LARGE', $R^{2}$ as 'very LARGE', $R^{4}$ as 'very very LARGE', and $\left(R^{2}\right)^{c}$ as 'not very LARGE'. To characterize linguistic hedges, a PF correlation measure $C$ must meet the following conditions.

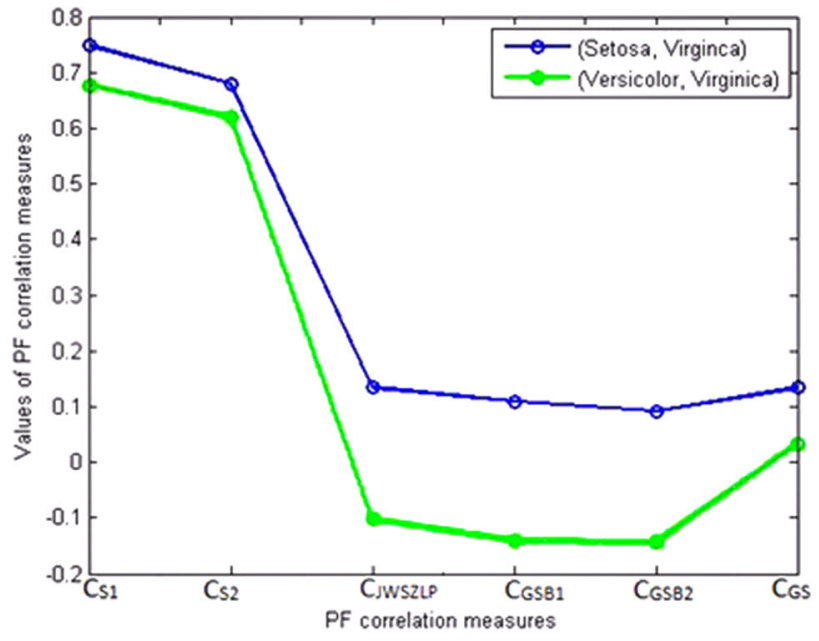

Fig. 3 Correlation between different classes of Iris plant

$C$ (M.L.L.,L.) $>C$ (M.L.L., V.L. $)>C($ M.L.L., V.V.L. $)$

$C($ V.V.L,V.L. $)>C($ V.V.L., L. $)>C($ V.V.L., M.L.L. $)$

$C($ L.,M.L.L. $)>C($ L., V.L. $)>C($ L., V.V.L. $)$

$C$ (V.L.,L.) $>C$ (V.L., V.V.L.) $>C$ (V.L., M.L.L.),

The computed values of the correlation between the linguistic hedges L., V.L., V.V.L., N.V.L., and M.L.L. using various PF correlation measures are given in Table 1.

From Table 1, we see that the existing PF correlation measures given in Eqs. (1)-(5) fail to satisfy one or more requirements given in Eqs. (8)-(11), whereas the suggested
Table 5 Calculated values of various $\mathrm{PF}$ correlation measures regarding Example 12

\begin{tabular}{llc}
\hline & $($ Setosa, Virginica $)$ & (Versicolor, Virginica) \\
\hline$C_{S 1}($ Singh 2015) & $\mathbf{0 . 7 4 8 8}$ & 0.6761 \\
$C_{S 2}($ Singh 2015) & $\mathbf{0 . 6 8 0 0}$ & 0.6201 \\
$C_{\mathrm{JWSZLP}}($ Jin et al. 2019) & $\mathbf{0 . 1 3 3 7}$ & -0.1034 \\
$C_{\mathrm{GSB} 1}($ Ganie et al. 2020) & $\mathbf{0 . 1 0 8 7}$ & -0.1405 \\
$C_{\mathrm{GSB} 2}($ Ganie et al. 2020) & $\mathbf{0 . 0 9 1 1}$ & -0.1434 \\
$C_{\mathrm{GS}}($ Proposed $)$ & $\mathbf{0 . 1 3 3 4}$ & 0.0333 \\
\hline
\end{tabular}

Bold values indicate maximum correlation 
PF correlation coefficient $C_{\mathrm{GS}}$ given in Eq. (6) satisfy all the conditions given in Eqs. (8)-(11). This indicates that the proposed correlation coefficient $C_{\mathrm{GS}}$ is more effective than the existing PF correlation coefficients in handling the linguistic hedges.

In the next section, we establish the rationality of our proposed correlation coefficient by showing its application in pattern recognition and decision-making.

\section{Applications}

In this section, we demonstrate the application of our proposed PF correlation measures in pattern recognition and decision-making and compare the results with some existing PF compatibility measures.

\subsection{Pattern recognition}

The main goal of pattern recognition is to determine how closely an unknown pattern resembles any previously recognized patterns. We use current PF correlation measures, as well as our own proposed PF correlation measure, to accomplish this. We begin by posing the pattern recognition problem in a $\mathrm{PF}$ context.

Scenario We are given some known patterns $V_{1}, V_{2}, \ldots, V_{k}$ and an unknown pattern $W$ expressed in the form of PFSs in $Y$ as.

$V_{j}=\left\{\left(y_{i}, \theta_{V_{j}}\left(y_{i}\right), \delta_{V_{j}}\left(y_{i}\right), \tau_{V_{j}}\left(y_{i}\right)\right) \mid y_{i} \in Y\right\}, j=1,2, \ldots, k$ and $W=\left\{\left(y_{i}, \theta_{W}\left(y_{i}\right), \delta_{W}\left(y_{i}\right), \tau_{W}\left(y_{i}\right)\right) \mid y_{i} \in Y\right\}$.

Aim To classify the unknown pattern $W$ into one of the known patterns $V_{j}(j=1,2, \ldots, k)$.

Recognition principle The unknown pattern $W$ can be assigned to the known pattern $V_{j}, j=1,2, \ldots, k$ with which it has a maximum correlation.

After determining the known pattern to which the unknown pattern belongs, we calculate the "Degree of Confidence (DoC)" (Hatzimichailidis et al. 2012) of each
PF-correlation measure, and this performance index measures the confidence of each PF-correlation measure in recognizing a specific sample that belongs to pattern $(i)$. The DoC is calculated as

$\operatorname{DoC}^{(i)}=\sum_{j=1, j \neq i}^{k}\left|K\left(V_{j}, W\right)-K\left(V_{i}, W\right)\right|$,

where $K$ is any PF-correlation measure. The greater the $\mathrm{DoC}^{(i)}$, the more confident the measure is. Now, we solve some pattern recognition problems involving PF information with the help of our proposed PF-correlation measure given in the Eq. (6) and compare the results with the existing PF-correlation measures given in the Eqs. (1)-(5).

Example 9 (Luo and Zhang 2020) Consider three known patterns $V_{1}, V_{2}$, and $V_{3}$ and an unknown pattern $W$ in the form of PFSs as.

$$
\begin{aligned}
& V_{1}=\left\{\left(y_{1}, 0.5,0.1,0.1\right),\left(y_{2}, 0.3,0.1,0.3\right),\left(y_{3}, 0.3,0.1,0.4\right)\right\}, \\
& V_{2}=\left\{\left(y_{1}, 0.4,0.3,0.2\right),\left(y_{2}, 0.3,0.2,0.5\right),\left(y_{3}, 0.4,0.1,0.3\right)\right\}, \\
& V_{3}=\left\{\left(y_{1}, 0.2,0.4,0.3\right),\left(y_{2}, 0.1,0.4,0.4\right),\left(y_{3}, 0.0,0.0,0.0\right)\right\} \text { and } \\
& W=\left\{\left(y_{1}, 0.3,0.2,0.3\right),\left(y_{2}, 0.4,0.1,0.3\right),\left(y_{3}, 0.4,0.2,0.3\right)\right\} .
\end{aligned}
$$

The calculated values of PF-correlation measures between the known patterns $V_{j}, j=1,2,3$, and the unknown pattern $W$ are summarized in Table 2 .

From Table 2, we see that except the correlation measures $C_{\text {JWSZLP }}$ and $C_{\mathrm{GSB} 1}$ all other PF correlation coefficients classify the unknown pattern $W$ into one of the known patterns $V_{j}, j=1,2,3$. The result of our proposed PF correlation coefficient $C_{\mathrm{GS}}$ is the same as that of $C_{\mathrm{GSB} 1}$ (Ganie et al. 2020). But the DoC of our proposed measure is very high than all the existing PF correlation measures and therefore is more effective. This indicates that the suggested PF correlation coefficient $C_{\mathrm{GS}}$ is more confident than all the existing PF correlation coefficients in assigning the unknown pattern $W$ to the known pattern $V_{1}$.
Table 6 PF representation of different masks

\begin{tabular}{lllll}
\hline Type of mask & $A_{1}$ & $A_{2}$ & $A_{3}$ & $A_{4}$ \\
\hline$P_{1}$ & $(0.01,0.69,0.06)$ & $(0.10,0.29,0.07)$ & $(0.18,0.12,0.13)$ & $(0.36,0.25,0.36)$ \\
$P_{2}$ & $(0.28,0.05,0.08)$ & $(0.20,0.35,0.43)$ & $(0.53,0.21,0.19)$ & $(0.36,0.28,0.39)$ \\
$P_{3}$ & $(0.15,0.36,0.40)$ & $(0.11,0.38,0.18)$ & $(0.20,0.11,0.09)$ & $(0.05,0.24,0.37)$ \\
$P_{4}$ & $(0.29,0.24,0.24)$ & $(0.19,0.41,0.31)$ & $(0.37,0.21,0.29)$ & $(0.53,0.07,0.17)$ \\
$P_{5}$ & $(0.08,0.15,0.37)$ & $(0.29,0.34,0.39)$ & $(0.07,0.46,0.46)$ & $(0.09,0.10,0.39)$ \\
$P_{6}$ & $(0.53,0.30,0.08)$ & $(0.64,0.33,0.01)$ & $(0.07,0.45,0.13)$ & $(0.7,0.24,0.46)$ \\
\hline
\end{tabular}


Table 7 Correlation of each mask with positive ideal PFS and negative ideal PFS

\begin{tabular}{lllllll}
\hline & $P_{1}$ & $P_{2}$ & $P_{3}$ & $P_{4}$ & $P_{5}$ & $P_{6}$ \\
\hline$C_{\mathrm{GS}}\left(P_{i}, P^{+}\right)$ & -0.3893 & 0.7009 & -0.2597 & 0.7377 & 0.6164 & 0.4021 \\
$C_{\mathrm{GS}}\left(P_{i}, P^{-}\right)$ & -0.4508 & 0.5035 & -0.3302 & 0.6442 & 0.5880 & 0.1738 \\
\hline
\end{tabular}

Table 8 Index of correlation

\begin{tabular}{lllllll}
\hline Correlation coefficient & $P_{1}$ & $P_{2}$ & $P_{3}$ & $P_{4}$ & $P_{5}$ & $P_{6}$ \\
\hline$C_{S 1}$ (Singh 2015) & 0.4909 & 0.5113 & 0.4676 & 0.5160 & 0.4523 & 0.5323 \\
$C_{S 2}$ (Singh 2015) & 0.4940 & 0.5067 & 0.4652 & 0.5105 & 0.4493 & 0.5314 \\
$C_{\text {JWSZLP }}$ (Jin et al. 2019) & 0.5457 & 0.4523 & 0.5581 & 0.4321 & 0.4807 & 0.5509 \\
$C_{\mathrm{GSB} 1}$ (Ganie et al. 2020) & 0.4423 & 0.6199 & 0.5191 & 0.4760 & 0.5675 & 0.5886 \\
$\mathrm{C}_{\mathrm{GSB} 2}$ (Ganie et al. 2020) & 0.5400 & 0.4301 & 0.5540 & 0.4023 & 0.4845 & 0.5572 \\
$C_{\mathrm{GS}}$ (Proposed) & 0.5265 & 0.5308 & 0.5250 & 0.5138 & 0.5044 & 0.5443 \\
\hline
\end{tabular}

Table 9 Ranking order of masks

\begin{tabular}{ll}
\hline Correlation Coefficient & Ranking \\
\hline$C_{S 1}$ (Singh 2015) & $P_{6}>P_{4}>P_{2}>P_{1}>P_{3}>P_{5}$ \\
$C_{S 2}$ (Singh 2015) & $P_{6}>P_{4}>P_{2}>P_{1}>P_{3}>P_{5}$ \\
$C_{\text {JWSZLP }}$ (Jin et al. 2019) & $P_{3}>P_{6}>P_{1}>P_{5}>P_{2}>P_{4}$ \\
$C_{\mathrm{GSB} 1}($ Ganie et al. 2020) & $P_{2}>P_{6}>P_{5}>P_{3}>P_{4}>P_{1}$ \\
$C_{\mathrm{GSB} 2}$ (Ganie et al. 2020) & $P_{6}>P_{3}>P_{1}>P_{5}>P_{2}>P_{4}$ \\
$C_{\mathrm{GS}}$ (Proposed) & $P_{6}>P_{2}>P_{1}>P_{3}>P_{4}>P_{5}$ \\
\hline
\end{tabular}

Example 10 (Luo and Zhang 2020) Consider three known patterns $V_{1}, V_{2}$, and $V_{3}$ and an unknown pattern $W$ in the form of PFSs as

$V_{1}=\left\{\left(y_{1}, 0.4,0.5,0.1\right),\left(y_{2}, 0.7,0.1,0.1\right),\left(y_{3}, 0.3,0.3,0.2\right)\right\}$,

$V_{2}=\left\{\left(y_{1}, 0.5,0.4,0.0\right),\left(y_{2}, 0.7,0.2,0.1\right),\left(y_{3}, 0.4,0.3,0.2\right)\right\}$,

$V_{3}=\left\{\left(y_{1}, 0.4,0.4,0.1\right),\left(y_{2}, 0.6,0.1,0.1\right),\left(y_{3}, 0.4,0.1,0.4\right)\right\}$ and

$W=\left\{\left(y_{1}, 0.1,0.1,0.6\right),\left(y_{2}, 0.7,0.1,0.2\right),\left(y_{3}, 0.8,0.1,0.1\right)\right\}$.

The calculated values of PF-correlation measures between the known patterns $V_{j}, j=1,2,3$, and the unknown pattern $W$ are summarized in Table 3 and also shown in Fig. 1.

From Table 3, it is clear that all the PF correlation measures assign the unknown pattern $W$ to one of the known patterns $V_{j}, j=1,2,3$. The result of our proposed PF correlation coefficient $C_{\mathrm{GS}}$ is the same as that of $C_{S 1}$ (Singh 2015) and $C_{\text {JWSZLP }}$ (Jin et al. 2019). However, the DoC of our proposed PF correlation coefficient $C_{\mathrm{GS}}$ is quite high than all the existing PF correlation measures as shown in Fig. 2. This indicates that the suggested PF correlation coefficient $C_{\mathrm{GS}}$ is more confident than all the existing PF correlation coefficients in assigning the unknown pattern $W$ to the known pattern $V_{2}$.

Example 11 Consider three known patterns $V_{1}, V_{2}$, and $V_{3}$ and an unknown pattern $W$ in the form of PFSs as.

$$
\begin{gathered}
V_{1}=\left\{\left(y_{1}, 0.5,0.0,0.3\right),\left(y_{2}, 0.7,0.0,0.0\right),\right. \\
\left.\left(y_{3}, 0.4,0.0,0.5\right),\left(y_{4}, 0.7,0.0,0.3\right)\right\}, \\
V_{2}=\left\{\left(y_{1}, 0.5,0.0,0.2\right),\left(y_{2}, 0.6,0.0,0.1\right),\right. \\
\left.\left(y_{3}, 0.2,0.0,0.7\right),\left(y_{4}, 0.7,0.0,0.3\right)\right\}, \\
V_{3}=\left\{\left(y_{1}, 0.5,0.0,0.4\right),\left(y_{2}, 0.7,0.0,0.1\right),\right. \\
\left.\left(y_{3}, 0.4,0.0,0.6\right),\left(y_{4}, 0.7,0.0,0.2\right)\right\} \text { and } \\
W=\left\{\left(y_{1}, 0.4,0.0,0.3\right),\left(y_{2}, 0.7,0.0,0.1\right),\right. \\
\left.\left(y_{3}, 0.3,0.0,0.6\right),\left(y_{4}, 0.7,0.0,0.3\right)\right\} .
\end{gathered}
$$

The calculated values of PF-correlation measures between the known patterns $V_{j}, j=1,2,3$, and the unknown pattern $W$ are summarized in Table 4 .

From Table 4, we see that only the PF correlation measures $C_{\mathrm{GSB} 1}$ and $C_{\mathrm{GS}}$ classify the unknown pattern $W$ to one of the known patterns $V_{i}, i=1,2,3$, and all other PF correlation measures fail to give satisfactory results. Also, the DoC of our proposed PF correlation coefficient $C_{\mathrm{GS}}$ is quite high and therefore is more confident than all of the existing PF correlation coefficients in assigning the unknown pattern $W$ to the known pattern $V_{2}$.

Thus, from Examples 8 to 10, we conclude that our proposed PF correlation coefficient is consistent with the existing PF correlation coefficients (Example 9) and in many cases (Examples 8 and 10) outperforms all of the existing PF correlation measures. Also, the DoC of the proposed PF correlation coefficient $C_{\mathrm{GS}}$ is very high than 
all of the existing PF correlation coefficients and therefore is more reasonable and effective.

Further, we apply our proposed PF correlation measure on the real data related to the Iris plant that has been obtained from the UCI Machine Learning Repository (https://archive.ics.uci.edu/ml/datasets/Iris).

Example 12 (Singh and Ganie 2021; Ganie and Singh 2021) The Iris plant database has 150 samples which are categorized into 3 classes "Setosa", "Versicolor", and "Virginica". Each sample has four features Petal Width (PW), Petal Length (PL), Sepal Width (SW), Sepal Length (SL). We compute the correlation between "Setosa" and "Virginica" and between "Versicolor" and "Virginica" using the suggested correlation coefficient for PFSs and contrast the performance with the existing correlation coefficient for PFSs. As the data in the Iris database is in the crisp form, so recently Singh and Ganie (2021) introduced a method for converting the data in the crisp form to the data in picture fuzzy form. The values of correlation between "Setosa" and "Virginica" and between "Versicolor" and "Virginica" are given in Table 5 and also the graphical representation is shown in Fig. 3.

From Table 5, we see that "Virginica" has maximum resemblance with "Setosa" as indicated by all the existing PF correlation measures. Our proposed PF correlation coefficient $C_{G S}$ also shows that Virginica has maximum resemblance with Setosa.

Now, we discuss the application of the proposed PF correlation measure in MADM.

\subsection{MADM}

In this section, we establish that our proposed PF correlation measure can be used in solving a MADM problem related to the selection of the best COVID-19 mask out of the available masks in the market. In a MADM problem, we are given a set of alternatives, a set of attributes and we want to find the best alternative. Here we solve a MADM problem concerning the selection of most suitable COVID19 mask.

Coronavirus Disease (COVID-19) has now become a global pandemic. This virus has affected the whole world and numerous people have lost their lives due to this deadly virus. Coronavirus is derived from the Latin word "corona" meaning a "crown, nimbus or circle of light". This virus immediately affects the lungs of a person. Cough, shortness of breath, and fever are its common symptoms. Spreading of this virus occurs through close contact and by the respiratory droplets from sneezes and coughs. The virus was first detected in the Wuhan City of China and later on, it engulfed the whole world. Many authors Chen et al. (2020), Lin et al. (2020), Zhao et al. (2020) have studied this virus from a mathematical point of view. Here, we try to find an appropriate COVID-19 mask out of some available masks.

Example 13 Gas masks $\left(P_{1}\right)$, medical protective masks $\left(P_{2}\right)$, ordinary nonmedical masks $\left(P_{3}\right)$, particulate respirators $(N 95)\left(P_{4}\right)$, medical-surgical masks $\left(P_{5}\right)$, and disposable medical masks $\left(P_{6}\right)$ are the six types of masks commonly available in the market. A customer would like to choose a suitable mask from the aforementioned masks based on four characteristics: $\left(A_{1}\right)$ filtration capability, $\left(A_{2}\right)$ quality of raw material, $\left(A_{3}\right)$ reusability, $\left(A_{4}\right)$ leakage rate. PFSs are used to represent people's opinions on the attributes related to each mask, as shown in Table 6. The positive ideal PFS $\left(P^{+}\right)$and the negative ideal PFS $\left(P^{-}\right)$ are calculated as:

$P^{+}=\left\{\left(\theta_{P^{+}}\left(y_{i}\right), \delta_{P^{+}}\left(y_{i}\right), \tau_{P^{+}}\left(y_{i}\right)\right) \mid y_{i} \in Y\right\}, P^{-}$

$=\left\{\left(\theta_{P^{-}}\left(y_{i}\right), \delta_{P^{-}}\left(y_{i}\right), \tau_{P^{-}}\left(y_{i}\right)\right) \mid y_{i} \in Y\right\}$,

where, $\theta_{P^{+}}\left(y_{i}\right)=\max \left\{\theta_{A_{j}}\left(y_{i}\right)\right\}, \quad \delta_{P^{+}}\left(y_{i}\right)=\min \left\{\delta_{A_{j}}\left(y_{i}\right)\right\}$, $\tau_{P^{+}}\left(y_{i}\right)=\min _{j}\left\{\tau_{A_{j}}\left(y_{i}^{j}\right)\right\}, \quad \theta_{P^{-}}\left(y_{i}\right)=\min _{j}\left\{\theta_{A_{j}}\left(y_{i}\right)\right\}, \quad \delta_{P^{-}}$ $\left(y_{i}\right)=\min _{j}\left\{\delta_{A_{j}}\left(y_{i}\right)\right\}$, and $\tau_{P^{-}}\left(y_{i}\right)=\max _{j}\left\{\tau_{A_{j}}\left(y_{i}\right)\right\}$.

From Table 6, we obtain the values of $P^{+}$and $P^{-}$as. $P^{+}=\{(0.53,0.05,0.06),(0.64,0.29,0.01)$,

$(0.53,0.11,0.09),(0.53,0.07,0.17)\}$, $P^{-}=\{(0.01,0.15,0.40),(0.10,0.29,0.43)$,

$(0.53,0.11,0.09),(0.53,0.07,0.17)\}$.

Next, we calculate the correlation of each $P_{i}, i=$ 1, 2, 3, 4, 5, 6 with $P^{+}$and $P^{-}$using our proposed PF correlation coefficient. The results are listed in Table 7 . To obtain the appropriate mask, we rank them in the descending order of the index of correlation $\xi_{i}$ given as

$\xi_{i}=\frac{1+C_{\mathrm{GS}}\left(P_{i}, P^{+}\right)}{2+C_{\mathrm{GS}}\left(P_{i}, P^{+}\right)+C_{\mathrm{GS}}\left(P_{i}, P^{-}\right)}, i=1,2,3,4,5,6$.

Table 8 shows the index of correlation for various masks. The ranking of masks is given in Table 9. Also, the ranking of alternatives by utilizing some existing $\mathrm{PF}$ correlation coefficients is given in the same Table 9.

From Table 9, we see that $\left(P_{6}\right)$, i.e., the disposable medical mask is the most appropriate mask as indicated by most of the PF correlation measures. Also, we see that the ranking pattern by different $\mathrm{PF}$ correlation coefficients is different. Our proposed PF correlation coefficient $C_{\mathrm{GS}}$ also shows that $\left(P_{6}\right)$, i.e., the disposable medical mask is the most appropriate mask. 


\section{Conclusion}

Although most correlation coefficients in fuzzy/non-standard fuzzy theory obtain their values in $[0,1]$, the proposed picture fuzzy correlation coefficient can compute both the degree of association and the nature of correlation (positive or negative). As a result, our correlation coefficient is similar to the traditional correlation coefficients used in statistics. With the help of linguistic hedges and computation of correlation degree, we have established the rationality of the suggested PF correlation coefficient. We have also demonstrated the use of our proposed correlation coefficient in pattern recognition with the aid of illustrative examples. A novel index called "Degree of Confidence" was also used to evaluate the efficiency of the proposed correlation coefficient. The proposed correlation coefficient has outperformed current correlation measures in terms of "Degree of Confidence." With a real data set related to the Iris plant (https://archive.ics.uci.edu/ml/datasets/Iris), the proposed PF correlation measure produced reliable results in a pattern classification problem. The MADM problem of selecting an appropriate COVID-19 mask has also been addressed using our proposed PF correlation measure. In the future, we shall derive some parametric generalizations of the PF correlation coefficients considered in this study along with their interdisciplinary applications. Moreover, the fusion of such correlation coefficients in the natureinspired algorithms also appears to provide fruitful solutions in optimization problems. We will also apply the proposed PF correlation coefficient to the real data concerning COVID-19.

Acknowledgements Authors are highly thankful to the anonymous reviewers and the Editor for their constructive suggestions and for bringing the paper in the present form.

Data availability All data generated or analysed during this study are included in this published article.

\section{Declarations}

Conflict of interest Authors declare that there is no conflict of interest.

\section{References}

Atanassov K (1986) Intuitionistic fuzzy sets. Fuzzy Sets Syst 20:87-96

Atanassov K, Gargov G (1989) Interval-valued intuitionistic fuzzy sets. Fuzzy Sets Syst 31:343-349

Bonizzoni P, Vedova GD, Dondi R, Jiang T (2008) Correlation clustering and consensus clustering. Lect Notes Comput Sc 3827:226-235

Bustince H, Burillo P (1995) Correlation of interval-valued intuitionistic fuzzy sets. Fuzzy Sets Syst 74:237-244
Chen SM (1996) A fuzzy reasoning approach for rule-based systems based on fuzzy logics. IEEE Trans Syst Man Cy B 26(5):769-778

Chen SM, Chiou CH (2014) Multiattribute decision making based on interval-valued intuitionistic fuzzy sets, PSO techniques, and evidential reasoning methodology. IEEE Trans Fuzzy Syst 23(6): 1905-1916

Chen SM, Huang CM (2003) Generating weighted fuzzy rules from relational database systems for estimating null values using genetic algorithms. IEEE Trans Fuzzy Syst 11(4):495-506

Chen SM, Huang ZC (2017) Multiattribute decision making based on interval-valued intuitionistic fuzzy values and linear programming methodology. Inf Sci 381:341-351

Chen SM, Jong WT (1997) Fuzzy query translation for relational database systems. IEEE T Syst Man Cy B 27(4):714-721

Chen SM, Randyanto Y (2013) A novel similarity measure between intuitionistic fuzzy sets and its applications. Int $\mathrm{J}$ Pattern Recognit Artif Intell 27(07):1350021

Chen SM, Tan JM (1994) Handling multicriteria fuzzy decisionmaking problems based on vague set theory. Fuzzy Sets Syst 67:163-172

Chen S, Yeh M, Hsiao P (1995) A comparison of similarity measures of fuzzy values. Fuzzy Sets Syst 72:79-89

Chen N, Xu Z, Xia M (2013) Correlation coefficients of hesitant fuzzy sets and their applications to clustering analysis. Appl Math Model 37:2197-2211

Chen T, Rui J, Wang Q, Zhao Z, Cui JA, Yin L (2020) A mathematical model for simulating the transmission of Wuhan novel Coronavirus. bioRxiv. https://doi.org/10.1101/2020.01.19. 911669

Cheung CF, Li FL (2012) A quantitative correlation coefficient mining method for business intelligence in small and medium enterprises of trading business. Expert Syst Appl 39:6279-6291

Chu CH, Hung KC, Julian PA (2014) Complete pattern recognition approach under Atanassov's intuitionistic fuzzy sets. Knowl Based Syst 66:36-45

Cuong BC (2014) Picture fuzzy sets. J Comput Sci Cybern 30:409-420

Cuong BC, Kreinovich V (2013) Picture fuzzy sets-a new concept for computational intelligence problems. In: Proceedings of the Third World Congress on information and communication technologies WICT, pp 1-6

De SK, Biswas R, Roy AR (2000) Some operations on intuitionistic fuzzy sets. Fuzzy Sets Syst 114:477-484

De SK, Biswas R, Roy AR (2001) An application of intuitionistic fuzzy sets in medical diagnosis. Fuzzy Sets Syst 117:209-213

Deschrijver G, Kerre EE (2003) On the composition of intuitionistic fuzzy relations. Fuzzy Sets Syst 136:333-361

Ganie AH, Singh S (2021) An innovative picture fuzzy distance measure and novel multi-attribute decision-making method. Complex Intell Syst. https://doi.org/10.1007/s40747-020-002353

Ganie AH, Singh S, Bhatia PK (2020) Some new correlation coefficients of picture fuzzy sets with applications. Neural Comput Appl 32:12609-12625

Gerstenkorn T, Manko J (1991) Correlation of intuitionistic fuzzy sets. Fuzzy Set Syst 44:39-43

Hatzimichailidis AG, Papakostas GA, Kaburlasos VG (2012) A novel distance measure of intuitionistic fuzzy sets and its application to pattern recognition problems. Int J Intell Syst 27:396-409

Hong DH (1998) A note on correlation of interval-valued intuitionistic fuzzy sets. Fuzzy Sets Syst 95:113-117

Hong DH, Choi CH (2000) Multicriteria fuzzy decision-making problems based on vague set theory. Fuzzy Sets Syst 114:103-113 
Hong DH, Hwang SY (1995) Correlation of intuitionistic fuzzy sets in probability spaces. Fuzzy Sets Syst 75:77-81

Huang HL (2012) (T, S)-Based interval-valued intuitionistic fuzzy composition matrix and its application for clustering. Iran $\mathrm{J}$ Fuzzy Syst 9:7-19

Hung WL (2001) Using statistical viewpoint in developing correlation of intuitionistic fuzzy sets. Int J Uncertain Fuzz 9:509-516

Hung WL, Wu JW (2002) Correlation of intuitionistic fuzzy sets by centroid method. Inf Sci 144:219-225

Jin Y, Wu H, Sun D, Zeng S, Luo D, Peng BA (2019) Multi-attribute Pearson's picture fuzzy correlation-based decision-making method. Mathematics 7(10):999

Khan MJ, Kumam P, Deebani W, Kumam W, Shah Z (2020a) Biparametric distance and similarity measures of picture fuzzy sets and their applications in medical diagnosis. Egypt Inf J. https:// doi.org/10.1016/j.eij.2020.08.002

Khan MJ, Kumam P, Liu P, Kumam W, Rehman WH (2020b) An adjustable weighted soft discernibility matrix based on generalized picture fuzzy soft set snd its applications in decisionmaking. J Intell Fuzzy Systems 38(2):2103-2118

Kumar A, Singh P, Kaur P, Kaur A (2011) A new approach for ranking of LR type generalized fuzzy numbers. Expert Syst Appl 38:10906-10910

Lin Q, Zhao S, Gao D, Lou Y, Yang S, Musa SS, Wang MH, Cai Y, Wang W, Yang L, He DA (2020) Conceptual model for the outbreak of Coronavirus disease 2019 (COVID-19) in Wuhan, China with individual reaction and governmental action. Int $\mathbf{J}$ Infect Dis 93:211-216

Liu P, Chen SM, Wang Y (2020) Multiattribute group decision making based on intuitionistic fuzzy partitioned Maclaurin symmetric mean operators. Inform Sci 512:830-854

Luo M, Zhang Y (2020) A new similarity measure between picture fuzzy sets and its application. Eng Appl Artif Intell 96:103956

Manoj TV, Leena J, Soney RB (1998) Knowledge representation using fuzzy Petri nets-revisited. IEEE T Knowl Data Eng 10(4):666-667

Mitchell HB (2004) A correlation coefficient for intuitionistic fuzzy sets. Int J Intell Syst 19:483-490

Park DG, Kwun YC, Park JH, Park IY (2009) Correlation coefficient of interval-valued intuitionistic fuzzy sets and its application to multiple attribute group decision-making problems. Math Comput Model 50:1279-1293

Riegel HP, Kroger P, Schubert E, Zimek A (2008) A General framework for increasing the robustness of PCA-based correlation clustering algorithms. Lect Notes Comput Sc 5069:418-435

Singh P (2015) Correlation coefficients for picture fuzzy sets. J Intell Fuzzy Syst 28:591-604

Singh S, Ganie AH (2021) Applications of picture fuzzy similarity measures in pattern recognition, clustering, and MADM. Expert Syst Appl 168:114264

Singh S, Lalotra S (2018) Generalized correlation coefficients of the hesitant fuzzy sets and the hesitant fuzzy soft sets with application in group decision-making. J Intell Fuzzy Syst 35:3821-3833

Singh S, Lalotra S (2019) On generalized correlation coefficients of the hesitant fuzzy sets with their application to clustering analysis. Comput Applied Math 38:11

Singh S, Sharma S (2019) On some generalized correlation coefficients of the fuzzy sets and fuzzy soft sets with application in cleanliness ranking of public health centers. J Intell Fuzzy Syst 36:3671-3683

Singh S, Lalotra S, Sharma S (2019) Dual concepts in fuzzy theory: entropy and knowledge measure. Int J Intell Syst 34:1034-1059

Singh S, Sharma S, Ganie AH (2020) On generalized knowledge measure and generalized accuracy measure with applications to MADM and pattern recognition. Comput Appl Math 39(3):1-44

Szmidt E, Kacprzyk J (2004) A similarity measure for intuitionistic fuzzy sets and its application in supporting medical diagnostic reasoning. Lect Notes Artif Int 3070:388-393

Wang CY, Chen SM (2017) Multiple attribute decision making based on interval-valued intuitionistic fuzzy sets, linear programming methodology, and the extended TOPSIS method. Inform Sci 397:155-167

Wang C, Zhou X, Tu H, Tao S (2017) Some geometric aggregation operators based on picture fuzzy sets and their application in multiple attribute decision making. Ital J Pure Appl Math 37:477-492

Wei GW (2016) Picture fuzzy cross-entropy for multiple attribute decision-making problems J. Bus Econ Manag 17:491-502

Wei GW (2017) Some cosine similarity measures for picture fuzzy sets and their applications to strategic decision making. Informatica 28:547-564

Wei GW (2018) Some similarity measures for picture fuzzy sets and their applications. Iran J Fuzzy Syst 15:77-89

Wei GW, Gao H (2018) The generalized dice similarity measures for picture fuzzy sets and their applications. Informatica 29:107-124

Wei GW, Wang HJ, Lin R (2011) Application of correlation coefficient to interval-valued intuitionistic fuzzy multiple attribute decision-making with incomplete weight information. Knowl Inf Syst 26:337-349

Xu Z, Xia M (2011) On distance and correlation measures of hesitant fuzzy information. Int J Intell Syst 26:410-425

Yue SH, Wu T, Pan J, Wang H (2013) Fuzzy clustering-based ET image fusion. Inf Fusion 14:487-497

Zadeh LA (1965) Fuzzy sets. Inf Control 8:338-353

Zadeh LA (1972) Fuzzy set interpretation of linguistic hedges. Cybernetics 2:4-34

Zeng S, Chen SM, Kuo LW (2019) Multiattribute decision making based on novel score function of intuitionistic fuzzy values and modified VIKOR method. Inf Sci 488:76-92

Zeng S, Chen SM, Fan KY (2020) Interval-valued intuitionistic fuzzy multiple attribute decision making based on nonlinear programming methodology and TOPSIS method. Inf Sci 506:424-442

Zhang ZH, Yang JY, Ye YP, Zhang Q (2012) A type of score function on intuitionistic fuzzy sets with double parameters and its application to pattern recognition and medical diagnosis. Proc Eng 29:4336-4342

Zhao S, Lin Q, Ran J, Musa SS, Yang G, Wang W, Lou Y, Gao D, Yang L, He D, Wang MH (2020) Preliminary estimation of the basic reproduction number of novel coronavirus (2019-nCoV) in China, from 2019 to 2020: a data-driven analysis in the early phase of the outbreak. Int J Infect Dis 92:214-217. https://doi. org/10.1016/j.ijid.2020.01.050

Publisher's Note Springer Nature remains neutral with regard to jurisdictional claims in published maps and institutional affiliations. 